Pacific Journal of Mathematics

BOUNDED WANKEL FORMS WITH WEIGHTED NORMS AND 


\section{BOUNDED HANKEL FORMS WITH WEIGHTED NORMS AND LIFTING THEOREMS}

\section{TAKAHIKO NAKAZI}

Bounded Hankel forms with respect to weighted norms are studied. The Nehari's theorem about the norms of the classical Hankel forms is generalized. This is essentially a lifting theorem due to Cotlar and Sadosky. Moreover a theorem about the essential norms of Hankel forms is proved. This relates with a theorem of Adamjan, Arov and Krein in the special case and gives a new lifting theorem which has applications to weighted norm inequalities, and the F. and M. Riesz theorem.

1. Introduction. Let

$$
A[a, b]=\sum_{i=-\infty}^{\infty} \sum_{j=-\infty}^{\infty} A_{i j} a_{i} b_{j}
$$

where $a$ and $b$ are finite sequences. Then $A[a, b]$ is called a sesquilinear form in the variables $a$ and $b$.

Let $\mathscr{P}$ be the set of all trigonometric polynomials and $m$ the normalized Lebesgue measure on the unit circle $T$. If we put $u=$ $\sum_{j=-n}^{n} a_{j} z^{j}$ for $a=\left(\ldots, 0, a_{-n}, \ldots, a_{0}, a_{1}, \ldots, a_{n}, 0, \ldots\right)$ then $u$ belongs to $\mathscr{P}$ and $\int|u|^{2} d m=\sum_{j=-n}^{n}\left|a_{j}\right|^{2}$. Let

$$
A(u, v)=A[a, b]
$$

where $u=\sum_{j=-n}^{n} a_{j} z^{j}$ and $v=\sum_{j=-m}^{m} \bar{b}_{j} \bar{z}^{j}$. Then we say that $A(u, v)$ is a sesquilinear form on $\mathscr{P} \times \mathscr{P}$. It is clear that

$$
A\left(\beta_{1} u_{1}+\beta_{2} u_{2}, v\right)=\beta_{1} A\left(u_{1}, v\right)+\beta_{2} A\left(u_{2}, v\right)
$$

and

$$
A\left(u, \alpha_{1} v_{1}+\alpha_{2} v_{2}\right)=\bar{\alpha}_{1} A\left(u, v_{1}\right)+\bar{\alpha}_{2} A\left(u, v_{2}\right) .
$$

If $A_{i j}=\alpha(i+j)$ then $A(u, v)$ is called a Hankel form on $\mathscr{P} \times \mathscr{P}$ and we will write those forms $\varphi(u, v), \psi(u, v)$ or etc.

Let $\mathscr{P}_{+}=\{f \in \mathscr{P}: \hat{f}(j)=0$ if $j<0\}$ and $\mathscr{P}_{-}=\{f \in \mathscr{P}: \hat{f}(j)=0$ if $j \geq 0\}$. If $A$ is restricted to $\mathscr{P}_{+} \times \mathscr{P}_{-}$then the restriction of $A$ is called a sesquilinear form on $\mathscr{P}_{+} \times \mathscr{P}_{-}$. If $\varphi$ is a Hankel form on $\mathscr{P} \times \mathscr{P}$ then we will write

$$
H_{\varphi}=\text { the restriction of } \varphi \text { to } \mathscr{P}_{+} \times \mathscr{P}_{-}
$$

and $\varphi$ is called a symbol of $H_{\varphi}$. 
A sesquilinear form $A$ on $\mathscr{P} \times \mathscr{P}$ is said to be bounded if there exists a positive constant $\gamma$ such that $|A(u, v)| \leq \gamma$ if $\int|u|^{2} d m \leq 1$ and $\int|v|^{2} d m \leq 1$. We will generalize this definition. Let $\mu$ and $\nu$ be finite positive Borel measures on $T$. A sesquilinear form $A$ on $\mathscr{P} \times \mathscr{P}$ is said to be bounded w.r.t. $(\mu, \nu)$ if there exists a positive constant $\gamma$ such that

$$
|A(u, v)|^{2} \leq \gamma^{2} \int|u|^{2} d \mu \int|v|^{2} d \nu \quad(u, v \in \mathscr{P}) .
$$

The smallest number $\gamma$ for which the inequality above is refered to as the norm of the form $A$ and we will write $\gamma=\||A \|| \mid$, where the pair of measures is fixed. Similarly for the norm $\gamma$ of the form $A$ on $\mathscr{P}_{+} \times \mathscr{P}_{-}$we will write $\gamma=\|A\|$. When the form $A(u, v)$ is bounded on $\mathscr{P} \times \mathscr{P}$ w.r.t. $(\mu, \nu)$, it can be extended to a form on (the $L^{2}(\mu)$ closure of $\mathscr{P}) \times\left(\right.$ the $L^{2}(\nu)$-closure of $\left.\mathscr{P}\right)$. Then we will still write $A\left(u^{\prime}, v^{\prime}\right)$ for $u^{\prime}$ and $v^{\prime}$ in the closures. It is the same for the case of $\mathscr{P}_{+} \times \mathscr{P}_{-}$.

For $0<p \leq \infty \quad H^{p}=H^{p}(m)$ denotes the usual Hardy space, that is, the $L^{p}=L^{p}(m)$-closure of $\mathscr{P}_{+}$. $C$ denotes the set of all continuous functions on $T$. Then $H^{\infty}+C$ is the closure of $\bigcup_{n=1}^{\infty} \bar{z}^{n} H^{\infty}$ [9, Theorem 2].

Our program is as follows. In $\S 2$ we will give representations of bounded Hankel forms on $\mathscr{P} \times \mathscr{P}$. In $\S 3$ generalizing Nehari's theorem ([13], [15, p. 6]) we will calculate the norms of bounded Hankel forms on $\mathscr{P}_{+} \times \mathscr{P}_{-}$. This is, in fact, the lifting theorem of Cotlar and Sadosky [4] that appears as a corollary in $\S 6$. In $\S 4$ we will determine compact bounded Hankel forms on $\mathscr{P}_{+} \times \mathscr{P}_{-}$. This relates with Hartman's theorem [8] in a special case. In $\S 5$ we will give the distance between a given Hankel form and the set of all compact sesquilinear forms. In $\S 6$ as a result of the previous sections we will obtain a new lifting theorem which contains one due to Cotlar and Sadosky [4]. In $\S 7$ we will apply results in the previous sections to problems in weighted norm inequalities as in [3] and to get a quantitative F. and M. Riesz theorem [16].

2. Bounded Hankel forms on $\mathscr{P} \times \mathscr{P}$. For some pair $\mu$ and $\nu$ of finite positive Borel measures on $T$, there exist nonzero bounded sesquilinear forms w.r.t. $(\mu, \nu)$ but in Corollary 1 it is shown that no nonzero Hankel forms can exist.

Proposition 1. If $\varphi$ is a bounded Hankel form on $\mathscr{P} \times \mathscr{P}$ w.r.t. $(\mu, \nu)$ and $\||\varphi|\|=\gamma$ then the following are valid. 
(1) There exists a finite Borel measure $\lambda$ on $T$ such that

$$
\varphi(u, v)=\int u \bar{v} d \lambda \quad(u, v \in \mathscr{P})
$$

and

$$
|\lambda(E)| \leq \gamma|\mu(E)||\nu(E)|
$$

for any Borel set $E$ in $T$.

(2) If $\mu=\mu_{a}+\mu_{s}$ and $\nu=\nu_{a}+\nu_{s}$ are Lebesgue decompositions w.r.t. $\lambda$ then $\varphi$ can be assumed to be a bounded Hankel form on $\mathscr{P} \times \mathscr{P}$ with respect to $\left(\mu_{a}, \nu_{a}\right)$.

Proof. There exists a bounded linear operator $\Phi$ from $L^{2}(\mu)$ to $L^{2}(\nu)$ such that $\varphi(u, v)=\int(\Phi u) \bar{v} d \nu$. Since $\varphi\left(z^{i}, \bar{z}^{j}\right)=\varphi\left(1, z^{i+j}\right)$,

$$
\varphi(u, v)=\int u \bar{v} k d \nu \quad(u, v \in \mathscr{P})
$$

where $k=\Phi 1 \in L^{2}(\nu)$. Set $d \lambda=k d \nu$; then

$$
\left|\int u \bar{v} d \lambda\right|^{2} \leq \gamma^{2} \int|u|^{2} d \mu \int|v|^{2} d \nu
$$

for any $u \in L^{2}(\mu)$ and $v \in L^{2}(\nu)$, and hence (1) follows. There is a Borel set $E_{a}$ in $T$ with $\mu_{s}\left(E_{a}\right)=\nu_{s}\left(E_{a}\right)=0$ on which $\lambda$ is concentrated. Then $\chi_{E_{a}} \in L^{2}(\mu) \cap L^{2}(\nu)$ and so

$$
\left|\int u \bar{v} d \lambda\right|^{2} \leq \gamma^{2} \int|u|^{2} d \mu_{a} \int|v|^{2} d \nu_{a}
$$

for any $u \in L^{2}\left(\mu_{a}\right)=\chi_{E_{a}} L^{2}(\mu)$ and $v \in L^{2}\left(\nu_{a}\right)=\chi_{E_{a}} L^{2}(\nu)$. This implies (2).

COROllaRy 1. If $\varphi$ is a bounded Hankel form on $\mathscr{P} \times \mathscr{P}$ w.r.t. $(\mu, \nu)$, and $\mu$ and $\nu$ are mutually singular, then $\varphi \equiv 0$.

COROllaRy 2. If $\varphi$ is a bounded Hankel form on $\mathscr{P} \times \mathscr{P}$ w.r.t. $\left(w_{1} d m, w_{2} d m\right)$, then for some $k$ in $L^{\infty}$

$$
\varphi(u, v)=\int u \bar{v} k \sqrt{w_{1} w_{2}} d m \quad(u, v \in \mathscr{P}) .
$$

Conversely such $\varphi$ is bounded w.r.t. $\left(w_{1} d m, w_{2} d m\right)$. 
3. Bounded Hankel forms on $\mathscr{P}_{+} \times \mathscr{P}_{-}$. In this section we will give a generalization of Nehari's theorem (see [13], [15, p. 6]) which was proved in the case of $\mu=\nu=m$. For any Hankel form $\varphi$ on $\mathscr{P} \times \mathscr{P}$, if $H_{\varphi}$ is bounded on $\mathscr{P}_{+} \times \mathscr{P}_{-}$w.r.t. $(\mu, \nu)$ then there exists a finite Borel measure $\lambda$ on $T$ such that

$$
\varphi(u, v)=\int u \bar{v} d \lambda \quad\left(u \in \mathscr{P}_{+}, v \in \mathscr{P}_{-}\right) .
$$

The proof is similar to the proof of Proposition 1. Let $\lambda=\lambda_{a}+\lambda_{s}$, $\mu=\mu_{a}+\mu_{s}$ and $\nu=\nu_{a}+\nu_{s}$ be Lebesgue decompositions with respect to $m$. Put

$$
\varphi_{a}(u, v)=\int u \bar{v} d \lambda_{a} \text { and } \varphi_{s}(u, v)=\int u \bar{v} d \lambda_{s}
$$

for any $u, v$ in $\mathscr{P}$. Then $H_{\varphi_{a}}$ and $H_{\varphi_{s}}$ are bounded Hankel forms on $\mathscr{P}_{+} \times \mathscr{P}_{-}$w.r.t. $\left(\mu_{a}, \nu_{a}\right)$ and $\left(\mu_{s}, \nu_{s}\right)$, respectively. Moreover $\max \left(\left\|H_{\varphi_{a}}\right\|,\left\|H_{\varphi_{s}}\right\|\right)=\left\|H_{\varphi}\right\|$.

For set

$$
H^{2}(\mu)=\text { the } L^{2}(\mu) \text {-closure of } \mathscr{P}_{+} \text {. }
$$

Then $\bar{z} \bar{H}^{2}(\mu)$ is the $L^{2}(\mu)$-closure of $\mathscr{P}_{-}$. Suppose $E_{s}$ is a Borel set with $m\left(E_{s}\right)=0$ where $\mu_{s}$ and $\nu_{s}$ are concentrated on $E_{s}$, and $E_{a}$ is a Borel set with $m\left(E_{a}\right)=1$ where $\mu_{a}$ and $\nu_{a}$ are concentrated on $E_{a} . E_{a}$ can be chosen to be the complement of $E_{s}$ in $T$. Then both the characteristic functions $\chi_{E_{a}}$ and $\chi_{E_{s}}$ belong to $H^{2}(\mu) \cap \bar{z} \bar{H}^{2}(\nu)$. Moreover $H^{2}(\mu)=\chi_{E_{a}} H^{2}(\mu) \oplus \chi_{E_{s}} H^{2}(\mu)$, and $\chi_{E_{a}} H^{2}(\mu)=H^{2}\left(\mu_{a}\right)$ and $\chi_{E_{s}} H^{2}(\mu)=H^{2}\left(\mu_{s}\right)=L^{2}\left(\mu_{s}\right)$. This implies the above statement about $H_{\varphi_{a}}$ and $H_{\varphi_{s}}$.

To prove the generalized Nehari's theorem, we need the following lemma which will be used in later sections, too.

Lemma 1. Let $A$ be a bounded sesquilinear form on $\mathscr{P}_{+} \times \mathscr{P}_{-}$w.r.t. $\left(w_{1} d m, w_{2} d m\right)$ and $w_{j}=\left|h_{j}\right|^{2}$ for $j=1,2$ where both $h_{1}$ and $h_{2}$ are outer functions in $\mathrm{H}^{2}$. If we put

$$
B(f, g)=A\left(h_{1}^{-1} f, \bar{h}_{2}^{-1} g\right) \quad\left(f \in \mathscr{P}_{+}, g \in \mathscr{P}_{-}\right)
$$

then $B$ is a bounded sesquilinear form w.r.t. ( $m, m, \quad)$ and $\|B\|=$ $\|A\|$.

Proof. Let $\gamma=\|A\|$; then

$$
|A(f, g)|^{2} \leq \gamma^{2} \int|f|^{2}\left|h_{1}\right|^{2} d m \int|g|^{2}\left|h_{2}\right|^{2} d m
$$


for any $f \in \mathscr{P}_{+}$and $g \in \mathscr{P}_{-}$. For any $f \in \mathscr{P}_{+}$and $g \in \mathscr{P}_{-}$, set $F=h_{1} f$ and $G=\bar{h}_{2} g$. Then $F \in H^{2}$ and $G \in \bar{z} \bar{H}^{2}$. Hence

$$
\left|A\left(h_{1}^{-1} F, h_{2}^{-1} G\right)\right|^{2} \leq \gamma^{2} \int|F|^{2} d m \int|G|^{2} d m .
$$

Since both $h_{1}$ and $h_{2}$ are outer functions, we get the lemma.

The following theorem is a generalization of Nehari's theorem (cf. [15, Theorem 1.3]) but this is the lifting theorem of Cotlar and Sadosky in [4], with other notation. A new proof is given here (cf. [17]).

THEOREM 2. Let $\varphi$ be a Hankel form on $\mathscr{P} \times \mathscr{P}$. If $H_{\varphi}$ is bounded w.r.t. $(\mu, \nu)$ then there exists a Hankel form $\psi$ bounded w.r.t. $(\mu, \nu)$ on $\mathscr{P} \times \mathscr{P}$ such that

$$
H_{\psi}=H_{\varphi} \quad \text { and } \quad\|\psi\|\|=\| H_{\varphi} \| .
$$

Proof. Let $\gamma=\left\|H_{\varphi}\right\|$. By the remark above Lemma 1

$$
\left|\varphi_{s}(f, g)\right|^{2} \leq \gamma^{2} \int|f|^{2} d \mu_{s} \int|g|^{2} d \nu_{s}
$$

for all $f \in \mathscr{P}_{+}$and $g \in \mathscr{P}_{-}$. Since $H^{2}\left(\mu_{S}\right)=L^{2}\left(\mu_{S}\right)$, this implies that $\left\|\left|\varphi_{s}\right|\right\| \leq \gamma$. Now we will prove that there exists a bounded Hankel form $\psi_{a}$ with respect to $\left(\mu_{a}, \nu_{a}\right)$ such that

$$
H_{\psi_{a}}=H_{\varphi_{a}} \text { and }\left\|\psi_{a}\right\|\|=\| H_{\varphi_{a}} \| .
$$

Then setting $\psi=\psi_{a}+\varphi_{s}$, the theorem follows because $\varphi=\varphi_{a}+\varphi_{s}$ and $\max \left(\left\|H_{\varphi_{a}}\right\|,\left\|H_{\varphi_{s}}\right\|\right)=\left\|H_{\varphi}\right\|$. Let $d \mu_{a}=w_{1} d m$ and $d \nu_{a}=$ $w_{2} d m$.

Case I. $\log w_{1} \notin L^{1}$ or $\log w_{2} \notin L^{1}$. We may assume that $\log w_{1} \notin L^{1}$. By the remark above Lemma 1 ,

$$
\left|\varphi_{a}(f, g)\right|^{2} \leq \gamma^{2} \int|f|^{2} w_{1} d m \int|g|^{2} w_{2} d m \quad\left(f \in \mathscr{P}_{+}, g \in \mathscr{P}_{-}\right) .
$$

Since $\log w_{1} \notin L^{1}, H^{2}\left(w_{1} d m\right)=L^{2}\left(w_{1} d m\right)$ and hence for any $u \in \mathscr{P}$ and $g \in \mathscr{P}_{-}$

$$
\left|\varphi_{a}(u, g)\right|^{2} \leq \gamma^{2} \int|u|^{2} w_{1} d m \int|g|^{2} w_{2} d m .
$$

Fix any $n \in Z_{+}$. For any $u_{1} \in \mathscr{P}$ and $g_{1} \in z^{n} \mathscr{P}_{-}$, there exists $u \in \mathscr{P}$ and $g \in \mathscr{P}_{-}$such that $u_{1}=z^{n} u$ and $g_{1}=z^{n} g$. Hence

$$
\begin{aligned}
\left|\varphi_{a}\left(u_{1}, g_{1}\right)\right|^{2} & =\left|\varphi_{a}\left(z^{n} u, z^{n} g\right)\right|^{2}=\left|\varphi_{a}(u, g)\right|^{2} \\
& \leq \gamma^{2} \int\left|u_{1}\right|^{2} w_{1} d m \int\left|g_{1}\right|^{2} w_{2} d m
\end{aligned}
$$


By the same argument for any $u, v \in \mathscr{P}$

$$
\left|\varphi_{a}(u, v)\right|^{2} \leq \gamma^{2} \int|u|^{2} w_{1} d m \int|v|^{2} w_{2} d m .
$$

This implies that $\left\|\varphi_{a}\right\| \leq \gamma$. Put $\psi_{a}=\varphi_{a}$.

Case II. $\log w_{1} \in L^{1}$ and $\log w_{2} \in L^{1}$. There exist outer functions $h_{1}$ and $h_{2}$ in $H^{2}$ such that $w_{1}=\left|h_{1}\right|^{2}$ and $w_{2}=\left|h_{2}\right|^{2}$ (cf. [6, p. 53]). Let $d \lambda_{a}=w_{3} d m$. By Lemma 1

$$
\begin{aligned}
& \left|\int f \bar{g}\left(h_{1} h_{2}\right)^{-1} w_{3} d m\right|^{2} \\
& \quad \leq \gamma^{2} \int|f|^{2} d m \int|g|^{2} d m \quad\left(f \in \mathscr{P}_{+}, g \in \mathscr{P}_{-}\right) .
\end{aligned}
$$

Let $s=w_{3}\left(h_{1} h_{2}\right)^{-1}$; then by a duality argument there exists $l \in H^{\infty}$ such that $\|s+l\|_{\infty} \leq \gamma$. By Schwarz's lemma, this implies that

$$
\left|\int(s+l) u_{1} \bar{u}_{2} d m\right|^{2} \leq \gamma^{2} \int\left|u_{1}\right|^{2} d m \int\left|u_{2}\right|^{2} d m \quad\left(u_{1}, u_{2} \in \mathscr{P}\right) .
$$

Let $v_{1}=h_{1}^{-1} u_{1}$ and $v_{2}=\bar{h}_{2}^{-1} u_{2}$ for any $u_{1}, u_{2} \in \mathscr{P}$. Then $v_{1} \in$ $L^{2}\left(w_{1} d m\right)$ and $v_{2} \in L^{2}\left(w_{2} d m\right)$. Hence

$$
\begin{aligned}
& \left|\int v_{1} v_{2} w_{3} d m+\int v_{1} \bar{v}_{2}\left(l h_{1} h_{2}\right) d m\right|^{2} \\
& \leq \gamma^{2} \int\left|v_{1}\right|^{2} w_{1} d m \int\left|v_{2}\right|^{2} w_{2} d m .
\end{aligned}
$$

Since $h_{1}^{-1} \mathscr{P}$ and $h_{2}^{-1} \mathscr{P}$ are dense in $L^{2}\left(w_{1} d m\right)$ and $L^{2}\left(w_{2} d m\right)$, respectively, if we put

$$
\varphi_{0}(u, v)=\int\left(\operatorname{lh}_{1} h_{2}\right) u \bar{v} d m \quad(u, v \in \mathscr{P})
$$

then $\varphi_{0}$ is a bounded Hankel form on $\mathscr{P} \times \mathscr{P}$ w.r.t. $\left(w_{1} d m, w_{2} d m\right)$, $H_{\varphi_{0}} \equiv 0$ and $\left\|\varphi_{a}+\varphi_{0}\right\| \leq \gamma$. Put $\psi_{a}=\varphi_{a}+\varphi_{0}$.

Theorem 2 implies that $\left\|H_{\varphi}\right\|=\inf \left\{\left\|\varphi+\varphi_{0}\right\| \|: H_{\varphi_{0}} \equiv 0\right\}$.

In Theorem 2 if $d \mu=d \nu=d m$ then Nehari's theorem follows and if $d \mu=d \nu=w d m$ then the scalar version of a theorem of Page [9] follows.

4. Compact bounded Hankel forms on $\mathscr{P}_{+} \times \mathscr{P}_{-}$. The ideas of this section are closely related to those of [2]. In particular, the concept of compact form and Theorem 3 are in Theorem 1a in [2]. Let $A$ be a 
bounded sesquilinear form on $\mathscr{P}_{+} \times \mathscr{P}_{-}$w.r.t. $(\mu, \nu)$. We say that $A$ is compact if there exists a null decreasing sequence $\left\{\gamma_{n}\right\}$ such that

$$
\left|A\left(z^{n} f, g\right)\right|^{2} \leq \gamma_{n}^{2} \int|f|^{2} d \mu \int|g|^{2} d \nu \quad\left(f \in \mathscr{P}_{+}, g \in \mathscr{P}_{-}\right)
$$

and

$$
\left|A\left(f, \bar{z}^{n} g\right)\right|^{2} \leq \gamma_{n}^{2} \int|f|^{2} d \mu \int|g|^{2} d \nu \quad\left(f \in \mathscr{P}_{+}, g \in \mathscr{P}_{-}\right)
$$

for $n=1,2, \ldots$. When $\gamma_{n}=0$ and $\gamma_{n-1} \neq 0$ for some $n, A$ is called finite $n$. In this section we will give a generalization of Hartman's theorem [8] which was proved in the case of $\mu=\nu=m$ and describes compact Hankel forms. However Theorem 4 does not show Hartman's theorem (see Remark).

LEMMA 2. If $A$ is a nonzero compact (finite $n \neq 0$, resp.) sesquilinear form w.r.t. $(\mu, \nu)$ associated with $\left\{\gamma_{n}\right\}$, then it is a nonzero compact (finite $n \neq 0$, resp.) sesquilinear form w.r.t. $\left(w_{1} d m, w_{2} d m\right)$ associated with $\left\{\gamma_{n}\right\}$ where $d \mu / d m=w_{1}$ and $d \nu / d m=w_{2}$. Moreover both $\log w_{1}$ and $\log w_{2}$ are integrable.

Proof. Let $E_{a}$ and $E_{s}$ be Borel sets as in the remark before Lemma 1. Then $\chi_{E_{a}}$ and $\chi_{E_{s}}$ belong to $H^{2}(\mu) \cap \bar{z} \bar{H}^{2}(\nu)$. Hence for $n=$ $1,2, \ldots$

$$
\left|A\left(\chi_{E_{s}} z^{n} f, g\right)\right|^{2} \leq \gamma_{n}^{2} \int|f|^{2} d \mu_{s} \int|g|^{2} d \nu \quad\left(f \in \mathscr{P}_{+}, g \in \mathscr{P}_{-}\right)
$$

and

$$
\left|A\left(f, \chi_{E_{s}} \bar{z}^{n} g\right)\right|^{2} \leq \gamma_{n}^{2} \int|f|^{2} d \mu \int|g|^{2} d \nu_{s} \quad\left(f \in \mathscr{P}_{+}, g \in \mathscr{P}_{-}\right) .
$$

Since $H^{2}\left(\mu_{s}\right)=L^{2}\left(\mu_{s}\right)$ and $H^{2}\left(\nu_{s}\right)=L^{2}\left(\nu_{s}\right)$, for $n=1,2, \ldots$

$$
\left|A\left(\chi_{E_{s}} u, g\right)\right|^{2} \leq \gamma_{n}^{2} \int|u|^{2} d \mu_{s} \int|g|^{2} d \nu \quad\left(u \in \mathscr{P}, g \in \mathscr{P}_{-}\right)
$$

and

$$
\left|A\left(f, \chi_{E_{s}} v\right)\right|^{2} \leq \gamma_{n}^{2} \int|f|^{2} d \mu \int|v|^{2} d \nu_{s} \quad\left(f \in \mathscr{P}_{+}, v \in \mathscr{P}_{-}\right) .
$$

As $n \rightarrow \infty$, it follows that $A\left(\chi_{E_{s}} f, g\right)=A\left(f, \chi_{E_{s}} g\right)=0$ for all $f \in \mathscr{P}_{+}$and $g \in \mathscr{P}_{-}$. Hence $A\left(z^{n} f, g\right)=A\left(\chi_{E_{a}} z^{n} f, \chi_{E_{a}} g\right)$ and $A\left(f, \bar{z}^{n} g\right)=A\left(\chi_{E_{a}} f, \chi_{E_{a}} \bar{z}^{n} g\right)$. This implies that $A$ is a nonzero 
compact (finite $n \neq 0$, resp.) sesquilinear form w.r.t. $\left(w_{1} d m\right.$, $\left.w_{2} d m\right)$ associated with $\left\{\gamma_{n}\right\}$. If $\log w_{1} \notin L^{1}$ or $\log w_{2} \notin L^{1}$ then $H^{2}\left(w_{1} d m\right)=L^{2}\left(w_{1} d m\right)$ or $H^{2}\left(w_{2} d m\right)=L^{2}\left(w_{2} d m\right)$. By the same argument to the above, we can show that $A$ is a zero form. Thus the lemma follows.

THEOREM 3. Let $n$ be a nonnegative integer.

(1) $H_{\varphi}$ is finite $n=0$ if and only if there exists a function $h$ in $H^{1}$ such that $\varphi(f, g)=\int f \bar{g} h d m\left(f \in \mathscr{P}_{+}, g \in \mathscr{P}_{-}\right)$.

(2) When $n \neq 0, H_{\varphi}$ is finite $n$ if and only if there exists a function $h$ in $\bar{z}^{n} H^{1}$ and out of $H^{1}$ such that $\varphi(f, g)=\int f \bar{g} h d m(f \in$ $\left.\mathscr{P}_{+}, g \in \mathscr{P}_{-}\right)$.

Proof. (1) There exists a finite Borel measure $\lambda$ such that $\varphi(f, g)=$ $\int f \bar{g} d \lambda\left(f \in \mathscr{P}_{+}, g \in \mathscr{P}_{-}\right)$. If $H_{\varphi}$ is zero, by the proof of Lemma 2 $\varphi(f, g)=\varphi\left(\chi_{E_{a}} f, \chi_{E_{a}} g\right)$ and hence $\lambda$ is absolutely continuous w.r.t. $d m$. Let $d \lambda=h d m$; then $h d m$ annihilates $z \mathscr{P}_{+}$and so $h \in H^{1}$. The converse is clear.

(2) Let $H_{\varphi}$ be finite, $n \neq 0$. By Corollary 2, Theorem 2 and Lemma 2 , there exists a nonzero function $h$ in $L^{1}$ such that

$$
\varphi(f, g)=\int f \bar{g} h d m \quad\left(f \in \mathscr{P}_{+}, g \in \mathscr{P}_{-}\right) .
$$

Since $H_{\varphi}$ is finite, $n \neq 0$, by Lemma 2 there exist $\gamma_{1}, \gamma_{2}, \ldots, \gamma_{n}$ with $\gamma_{n}=0$ such that for $1 \leq j \leq n$

$$
\begin{aligned}
& \left|\int z^{j} f \bar{g} h d m\right|^{2} \\
& \quad \leq \gamma_{j}^{2} \int|f|^{2} w_{1} d m \int|g|^{2} w_{2} d m \quad\left(f \in \mathscr{P}_{+}, g \in \mathscr{P}_{-}\right),
\end{aligned}
$$

where $w_{1}=d \mu / d m$ and $w_{2}=d \nu / d m$. Moreover there exist outer functions $h_{1}$ and $h_{2}$ such that $\left|h_{j}\right|^{2}=w_{j}$ for $j=1,2$. By Lemma 1 , for $1 \leq j \leq n$

$$
\begin{aligned}
& \left|\int z^{j} f \bar{g}\left(h_{1} h_{2}\right)^{-1} h d m\right|^{2} \\
& \quad \leq \gamma_{j}^{2} \int|f|^{2} d m \int|g|^{2} d m \quad\left(f \in \mathscr{P}_{+}, g \in \mathscr{P}_{-}\right)
\end{aligned}
$$

and hence $\left\|z^{j}\left(h_{1} h_{2}\right)^{-1} h+H^{\infty}\right\| \leq \gamma_{j}$. Since $\gamma_{n}=0,\left(h_{1} h_{2}\right)^{-1} h \in$ $\bar{z}^{n} H^{\infty}$ and hence $h \in \bar{z}^{n} H^{1}$ and $h \notin H^{1}$ because $H_{\varphi}$ is rank $n \neq 0$. The converse is clear because for such $h, \int z^{n} f \bar{g} h d m=0 \quad(f \in$ $\left.\mathscr{P}_{+}, g \in \mathscr{P}_{-}\right)$. 
In the proof of Theorem 3, $h_{1} h_{2} \in H^{1}$ and $h=\left(h_{1} h_{2}\right) u$ where $u \in \bar{z}^{n} H^{\infty}$. The following theorem is the generalization of this result.

THEOREM 4. $H_{\varphi}$ is nonzero and compact w.r.t. $(\mu, \nu)$ if and only if there exists a function $h=h_{0} \times u$ in $H^{1} \times\left(H^{\infty}+C\right)$ and out of $H^{1}$ such that

$$
\varphi(f, g)=\int f \bar{g} h d m \quad\left(f \in \mathscr{P}_{+}, g \in \mathscr{P}_{-}\right)
$$

and $h_{0}=h_{1} h_{2}$ where $h_{j}$ is an outer function in $H^{2}, w_{j}=\left|h_{j}\right|$, $d \mu / d m=w_{1}$ and $d \nu / d m=w_{2}$.

Proof. Let $H_{\varphi}$ be nonzero and compact. By Lemma 2, we may assume that $d \mu=w_{1} d m$ and $d \nu=w_{2} d m$, and there exists an outer function $h_{j}$ in $H^{2}$ with $w_{j}=\left|h_{j}\right|^{2}$. By the proof of Theorem 3, $\left\|z^{j}\left(h_{1} h_{2}\right)^{-1} h+H^{\infty}\right\| \leq \gamma_{j}$ and $\gamma_{j} \rightarrow 0$ as $j \rightarrow \infty$. Thus $\left(h_{1} h_{2}\right)^{-1} h \in$ $H^{\infty}+C$ and hence $h=\left(h_{1} h_{2}\right) u \in H^{1} \times\left(H^{\infty}+C\right)$ and out of $H^{1}$. For the converse, put $\left\|z^{j} u+H^{\infty}\right\|=\gamma_{j}$; then $\gamma_{j} \rightarrow 0$ as $j \rightarrow \infty$ and for each $j$ there exists $g_{j} \in H^{\infty}$ such that

$$
\left|z^{j} h+h_{1} h_{2} g_{j}\right| \leq \gamma_{j}\left|h_{1} h_{2}\right| \text {. }
$$

Hence for each $j$

$$
\begin{aligned}
\left|\varphi\left(z^{j} f, g\right)\right|^{2} & =\left|\int z^{j} f \bar{g} h d m\right|^{2} \leq \gamma_{j}^{2} \int|f \bar{g}|\left|h_{1} h_{2}\right| d m \\
& \leq \gamma_{j}^{2} \int|f|^{2} w_{1} d m \int|g|^{2} w_{2} d m
\end{aligned}
$$

for all $f \in \mathscr{P}_{+}$and $g \in \mathscr{P}_{-}$. This implies that $H_{\varphi}$ is nonzero and compact w.r.t. $(\mu, \nu)$.

If $h=h_{0} \times u$ is in $H^{1} \times\left(H^{\infty}+C\right)$ and $\varphi_{1}(f, g)=\int f \bar{g} h d m$ $\left(f \in \mathscr{P}_{+}, g \in \mathscr{P}_{-}\right)$then $H_{\varphi_{1}}$ is compact w.r.t. $\left(\mu_{1}, \nu_{1}\right)$ where $d \mu_{1}=$ $d \nu_{1}=\left|h_{0}\right|^{2} d m$.

If $\mu$ is a complex finite Borel measure on $T$ and $\hat{\mu}(n)=\int e^{-i n \theta} d \mu$ $=0$ for any negative integer $n$, then $d \mu=h d m$ for some $h$ in $H^{1}$. This is the famous F. and M. Riesz theorem (cf. [11, p. 47]) and a corollary of the following corollary which follows from Theorem 3 and 4. That is, it is just the case of $\varepsilon_{0}=0$.

Corollary 4. Let $\mu$ be a complex finite Borel measure on $T$ and

$$
\varepsilon_{n}=\sup \left\{\left|\int z^{n} F d \mu\right| ; F \in \mathscr{P}_{+}, \int|F| d|\mu| \leq 1\right\} .
$$


If $\varepsilon_{n} \rightarrow 0$ as $n \rightarrow \infty$ then $\mu=h d m$ and $h$ is in $H^{1} \times\left(H^{\infty}+C\right)$. If $\varepsilon_{n}=0$ for some $n \geq 0$ then $h$ belongs to $\bar{z}^{n} H^{1}$.

Proof. By Schwarz's lemma,

$$
\begin{aligned}
\sup \left\{\left|\int z^{n} f \bar{g} d \mu\right| ; f \in \mathscr{P}_{+}, g \in \mathscr{P}_{-},\right. & \int|f|^{2} d|\mu| \leq 1 \\
& \text { and } \left.\int|g|^{2} d|\mu| \leq 1\right\} \leq \varepsilon_{n} .
\end{aligned}
$$

Now apply Theorems 3 and 4 for $\varphi\left(z^{n} f, g\right)=\int z^{n} f \bar{g} d \mu$.

5. Distance between $H_{\varphi}$ and the set of all compact sesquilinear forms.

THEOREM 5. Let $H_{\varphi}$ be a bounded Hankel form and $A$ a compact (finite $n$, resp.) sesquilinear form on $\mathscr{P}_{+} \times \mathscr{P}_{-}$w.r.t. $(\mu, \nu)$. If $\left\|H_{\varphi}+A\right\| \leq \gamma$ then there exists a symbol $\psi$ such that $H_{\psi}$ is a compact (finite $n$, resp.) Hankel form w.r.t. $(\mu, \nu)$ and $\|\varphi+\psi\| \mid \leq \gamma$.

Proof. By the remark preceding Lemma 1, we can decompose $\varphi=$ $\varphi_{a}+\varphi_{s}$ where $H_{\varphi_{a}}$ is bounded w.r.t. $\left(\mu_{a}, \nu_{a}\right)$ and $H_{\varphi_{s}}$ is bounded w.r.t. $\left(\mu_{s}, \nu_{s}\right)$. If $\left\|H_{\varphi}+A\right\| \leq \gamma$ then by Lemma 2 and the proof of Theorem $2\left\|\varphi_{s}\right\| \leq \leq$ and $\left\|H_{\varphi_{a}}+A\right\| \leq \gamma$. Hence we may assume that $\varphi=\varphi_{a}, \mu=\mu_{a}=w_{1} d m$ and $\nu=\nu_{a}=w_{2} d m$. If $\log w_{1} \notin L^{2}(m)$ or $\log w_{2} \notin L^{1}(m)$, by Lemma $2 A(f, g)=0 \quad\left(f \in \mathscr{P}_{+}, g \in \mathscr{P}_{-}\right)$ and hence Theorem 2 implies the theorem. By Lemma 1

$$
\begin{aligned}
\mid \varphi\left(h_{1}^{-1} f, \bar{h}_{2}^{-1} g\right) & +\left.A\left(h_{1}^{-1} f, h_{2}^{-1} g\right)\right|^{2} \\
& \leq \gamma^{2} \int|f|^{2} d m \int|g|^{2} d m \quad\left(f \in \mathscr{P}_{+}, g \in \mathscr{P}_{-}\right)
\end{aligned}
$$

and there exists a null decreasing sequence $\left\{\gamma_{n}\right\}$ such that

$$
\begin{aligned}
& \left|A\left(h_{1}^{-1} z^{n} f, h_{2}^{-1} g\right)\right|^{2} \\
& \quad \leq \gamma_{n}^{2} \int\left|z^{n} f\right|^{2} d m \int|g|^{2} d m \quad\left(f \in \mathscr{P}_{+}, g \in \mathscr{P}_{-}\right) .
\end{aligned}
$$

Hence there exist bounded linear operators $H_{l}$ and $\mathscr{A}$ from $H^{2}(m)$ to $\bar{z} \bar{H}^{2}(m)$ such that

$$
\left(H_{l} f, g\right)=(l f, g)=\varphi\left(h_{1}^{-1} f, h_{2}^{-1} g\right)
$$

and

$$
(\mathscr{A} f, g)=A\left(h_{1}^{-1} f, h_{2}^{-1} g\right)
$$


where $l \in L^{\infty}(m)$ and $($,$) denotes the usual inner product with$ respect to $m$. Let $U$ be a unilateral shift on $H^{2}$; then $\left\|\mathscr{A} U^{n}\right\| \rightarrow 0$ because $\gamma_{n} \rightarrow 0$. By the same argument as in $[10, p .6]$, there exists a function $k \in H^{\infty}+C$ such that $\|l+k\|_{\infty}<1$. Similarly to the proof of Theorem 2 put

$$
\psi(u, v)=\int\left(k h_{1} h_{2}\right) u \bar{v} d m \quad(u, v \in \mathscr{P}) .
$$

Then $\psi$ is a bounded Hankel form w.r.t. $\left(w_{1} d m, w_{2} d m\right)$ and by Theorem $4 H_{\psi}$ is compact. Thus $\|\varphi+\psi\| \| \leq \gamma$.

Theorem 5 implies that $\inf \left\{\left\|H_{\varphi}+A\right\|: A\right.$ ranges over all compact sesquilinear forms $\}=\inf \left\{\|\mid \varphi+\psi\| \|: H_{\psi}\right.$ ranges over all compact Hankel forms $\}$. When $d \mu=d \nu=d m$, this relates a theorem of Adamjan, Arov and Krein (cf. [1], [15, p. 6]). However the former does not imply the latter (see Remark).

6. Lifting theorem. In this section we obtain a new lifting theorem which contains one due to Cotlar and Sadosky [2]. Let $A_{i j} \quad(i, j=$ $1,2)$ be bilinear forms on $\mathscr{P} \times \mathscr{P}$ and suppose

$$
A_{11}(u, u) \geq 0, \quad A_{22}(u, u) \geq 0 \quad \text { and } \quad A_{12}(u, v)=\overline{A_{21}(u, v)} \text {. }
$$

Set

$$
\mathbf{A}(\mathbf{u}, \mathbf{u})=\sum_{i, j=1}^{2} A_{i j}\left(u_{i}, u_{j}\right)
$$

where $\mathbf{u}=\left(u_{1}, u_{2}\right)$ and $u_{i} \in \mathscr{P}$ for $i=1,2$. We write $\mathbf{A}=\left[A_{i j}\right]$. If $\rho_{i j}(i, j=1,2)$ are finite Borel measures on $T$ and

$$
A_{i j}(u, v)=\int u \bar{v} d \rho_{i j} \quad\left(u \in \mathscr{P}_{+}, v \in \mathscr{P}_{-}\right),
$$

then $A_{i j}(i, j=1,2)$ are bounded Hankel forms on $\mathscr{P} \times \mathscr{P}$ w.r.t. $\left(\left|\rho_{i j}\right|,\left|\rho_{i j}\right|\right)$. By the hypothesis on $\left[A_{i j}\right]$

$$
\rho_{11} \geq 0, \quad \rho_{22} \geq 0 \text { and } \rho_{12}=\bar{\rho}_{21} .
$$

We write $\mathbf{A}=\left[A_{i j}\right]=\left[\rho_{i j}\right]=\rho$ and we call $\rho$ a matrix of measures. $\mathbf{A} \succ 0$ w.r.t. $\Gamma$ means that $\mathbf{A}$ is positive w.r.t. $\Gamma$ :

$$
\mathbf{A}(\mathbf{u}, \mathbf{u})=\sum_{i, j=1}^{2} A_{i j}\left(u_{i}, u_{j}\right) \geq 0 \quad(\mathbf{u} \in \Gamma)
$$

where $\Gamma$ denotes $\mathscr{P} \times \mathscr{P}$ or $\mathscr{P}_{+} \times \mathscr{P}_{-}$.

We say that $\mathbf{A}$ is compact (finite $n$, resp.) w.r.t. $\rho$ if $A_{11}=A_{22}=$ 0 and $A_{12}$ is compact (finite $n$ ) w.r.t. $\left(\rho_{11}, \rho_{22}\right)$. 
THEOREM 6. Let $\boldsymbol{\rho}$ be a matrix of measures. If

$$
\boldsymbol{\rho}+\mathbf{A} \succ 0 \quad \text { w.r.t. } \mathscr{P}_{+} \times \mathscr{P}_{-}
$$

where $\mathbf{A}$ is compact (finite $n$, resp.) w.r.t. $\boldsymbol{\rho}$, then there exists a compact (finite $n$, resp.) matrix $\tau$ of measures w.r.t. $\boldsymbol{\rho}$ such that

$$
\boldsymbol{\rho}+\boldsymbol{\tau} \succ 0 \quad \text { w.r.t. } \mathscr{P} \times \mathscr{P} .
$$

Proof. Let

$$
\varphi_{12}(f, g)=\int f \bar{g} d \rho_{12} \quad\left(f \in \mathscr{P}_{+}, g \in \mathscr{P}_{-}\right) .
$$

Then $\varphi_{12}+A_{12}$ is a bounded bilinear form on $\mathscr{P}_{+} \times \mathscr{P}_{-}$w.r.t. $\left(\rho_{11}\right.$, $\left.\rho_{22}\right)$ because $\boldsymbol{\rho}+\mathbf{A} \succ 0$. Let $\left\|\varphi_{12}+A_{12}\right\| \leq \gamma$. By Theorem 5, there exists a symbol $\psi$ such that $H_{\psi}$ is a compact (finite $n$, resp.) w.r.t. $\left(\rho_{11}, \rho_{22}\right)$ and $\left\|\varphi_{12}+\psi\right\| \| \leq \gamma$. By Theorems 3 and 4 , there exists a function $h$ in $L^{1}$ such that

$$
\psi(f, g)=\int f \bar{g} h d m \quad\left(f \in \mathscr{P}_{+}, g \in \mathscr{P}_{-}\right) .
$$

Then $d \tau_{12}=h d m$ is the desired measure.

Corollary 3 (Cotlar and Sadosky). Let $\boldsymbol{\rho}$ be a matrix of measures. If

$$
\boldsymbol{\rho} \succ 0 \quad \text { w.r.t. } \mathscr{P}_{+} \times \mathscr{P}_{-}
$$

then there exists a finite $n=0$ matrix $\tau$ of measures such that

$$
\boldsymbol{\rho}+\boldsymbol{\tau} \succ 0 \quad \text { w.r.t. } \mathscr{P} \times \mathscr{P} .
$$

By Theorems 3 and 4, we can describe compact (finite $n$, resp.) matrices of measures w.r.t. $\rho$.

7. Weighted norm inequalities. In this section we show known results in the $L^{2}$ weighted problem, using the theorems of $\S \S 3,4$ and 5 . For any fixed nonnegative integer $n$, we want to find the positive measure $\mu$ for which there is a nonzero positive measure $\nu_{n}$ such that

$$
\int\left|z^{n} f\right|^{2} d \nu_{n} \leq \int\left|z^{n} f+g\right|^{2} d \mu \quad\left(f \in \mathscr{P}_{+}, g \in \mathscr{P}_{-}\right) .
$$

The inequality above is equivalent to the following one:

$$
\left|\int z^{n} f \bar{g} d \mu\right|^{2} \leq \int|f|^{2} d\left(\mu-\nu_{n}\right) \int|g|^{2} d \mu \quad\left(f \in \mathscr{P}_{+}, g \in \mathscr{P}_{-}\right) .
$$


Hence the problem is related with prediction problems when such a measure $\mu$ arises as the spectral density of a discrete weakly stationary Gaussian stochastic process. The following proposition is due to Arocena, Cotlar and Sadosky [3]. The Helson-Szegö theorem [10] and the Koosis theorem [12] follow from the first part in it.

Proposition 7. Let $\mu$ be a positive measure. There is a nonzero positive measure $\nu$ such that

$$
\int|f|^{2} d \nu \leq \int|f+g|^{2} d \mu \quad\left(f \in \mathscr{P}_{+}, g \in \mathscr{P}_{-}\right)
$$

if and only if $d \nu=u d m$ and there is a nonzero $k$ in $H^{1}$ such that

$$
|w+k|^{2} \leq(w-u) w
$$

where $d \mu=w d m+d \mu_{s}$. Then if $\log (w-u)$ is in $L^{1}$ then $u \leq$ $\left(1-\gamma^{-1}\right) w$ and $\gamma>1$.

We can prove Proposition 7 using the lifting theorem of Cotlar and Sadosky (Theorem 2 or Corollary 3) as that in [3]. The following theorem is closely related to results in [3]. We will give a proof using Theorems 3 and 4.

THEOREM 8. Let $\mu$ be a positive measure. For any fixed nonnegative integer $n$, let $\nu_{n}$ be a nonzero positive measure such that

$$
\int\left|z^{n} f\right|^{2} d \nu_{n} \leq \int\left|z^{n} f+g\right|^{2} d \mu \quad\left(f \in \mathscr{P}_{+}, g \in \mathscr{P}_{-}\right) .
$$

Suppose that there exists a positive measure $\lambda$ and a decreasing sequence $\left\{\varepsilon_{n}\right\}$ such that $\nu_{n}=\mu-\varepsilon_{n} \lambda$ and $0 \leq \varepsilon_{n} \leq 1$.

(1) $\varepsilon_{n}=0$ for some $n$ if and only if $d \nu_{n}=d \mu=w d m$ and $w=s h$ where $h$ is an outer function with $w=|h|$ and $s$ is in $\bar{z}^{n} H^{\infty}$.

(2) $\varepsilon_{n} \rightarrow 0$ as $n \rightarrow \infty$ if and only if $d \nu_{n}=\left(w_{1}-\varepsilon_{n} w_{2}\right) d m$, $d \mu=w_{1} d m, d \lambda=w_{2} d m+d \lambda_{s}$ and $w_{1}=s h_{1} h_{2}$ where $h_{j}$ is an outer function with $w_{j}=\left|h_{j}\right|^{2}$ for $j=1,2$ and $s$ is in $H^{\infty}+C$.

Proof. Set

$$
\varphi(u, v)=\int u \bar{v} d \mu \quad(u, v \in \mathscr{P})
$$

then by the remark before Theorem $7 H_{\varphi}$ is finite $n$ and compact w.r.t. $(\lambda, \mu)$ for (1) and (2), respectively. (1) follows from (2) of Theorem 3. For if $\varepsilon_{n}=0$ for some $n$ then $w \in \bar{z}^{n} H^{1}$ and hence $w=|h|=\bar{z}^{n} q h$ where $q$ is in $H^{\infty}$. (2) follows from Theorem 4 . 
In Theorem 8 , if $\lambda=\mu$ this was proved by Helson and Sarason [10]. Theorem 8 is also a corollary of Theorem 6 which is a new lifting theorem.

REMARK. Hankel operators from $H^{2}(\mu)$ to $\bar{z} \bar{H}^{2}(\nu)$. Let $\mu$ and $\nu$ be finite positive Borel measures on $T . M_{z}^{\mu}$ and $M_{z}^{\nu}$ are multiplication operators by the coordinate function $z$ on $L^{2}(\mu)$ and $L^{2}(\nu)$, respectively. Let $\Phi$ be a bounded linear operator from $L^{2}(\mu)$ to $L^{2}(\nu)$ and $(\boldsymbol{\Phi} u, v)=\int(\boldsymbol{\Phi} u) \bar{v} d \nu$ for $u, v$ in $\mathscr{P}$. Then $\boldsymbol{\Phi} M_{z}^{\mu}=M_{z}^{\nu} \boldsymbol{\Phi}$ if and only if $\varphi(u, v)=(\boldsymbol{\Phi} u, v)$ is a bounded Hankel form on $\mathscr{P} \times \mathscr{P}$ w.r.t. $(\mu, \nu)$. Let $P$ and $Q$ be the orthogonal projections from $L^{2}(\mu)$ to $H^{2}(\mu)$ and from $L^{2}(\nu)$ to $\bar{z} \bar{H}^{2}(\nu)$, respectively. Put $H=Q \Phi P$; then $(H f, g)=H_{\varphi}(f, g)$ for $f$ in $\mathscr{P}_{+}$ and $g$ in $\mathscr{P}_{-}$. Put $S_{z}^{\mu}=P M_{z}^{\mu} \mid H^{2}(\mu)$ and $S_{\bar{z}}^{\nu}=Q M \frac{\nu}{z} \mid \bar{z} \bar{H}^{2}(\nu)$; then $H S_{z}^{\mu}=\left(S_{\bar{z}}^{\nu}\right)^{*} H$. Theorem 2 calculates the norm of $H$. In general, even if $H$ is a compact linear operator, $H_{\varphi}$ may not be a compact sesquilinear form.

When $\boldsymbol{\mu}=\boldsymbol{\nu}=m, \boldsymbol{\Phi}$ is a multiplication operator $M_{\Phi}$ by a function $\Phi$ in $L^{\infty}(m)$ and $\|\Phi\|=\|\Phi\|_{\infty}=\|\| \varphi \| . \quad H$ is called a Hankel operator and $\|H\|=\left\|H_{\varphi}\right\| . H_{\varphi}$ is a compact Hankel form if and only if $H$ is a compact Hankel operator. For by Theorem $4 H_{\varphi}$ is compact if and only if $\varphi(f, g)=\int f \bar{g} h d m\left(f \in \mathscr{P}_{+}, f \in \mathscr{P}_{-}\right)$ and $h \in H^{\infty}+C$. By Hartman's theorem (cf. [15, Theorem 1.4]) $H$ is compact if and only if $\Phi \in H^{\infty}+C$. Moreover the essential norm $\|H\|_{e}$ of $H$ coincides with $\inf \left\{\left\|H_{\varphi}+A\right\|: A\right.$ ranges over all compact sesquilinear forms\}. For by a theorem of Adamjan, Arov and Krein [1], $\|H\|_{e}=\left\|\Phi+H^{\infty}+C\right\|$. While by Theorems 4 and $5 \inf \left\|H_{\varphi}+A\right\|=\inf \left\{\|\varphi+\psi\| \|: H_{\psi}\right.$ ranges over all compact Hankel forms $\}=\left\|h+H^{\infty}+C\right\|$ where $\varphi(f, g)=\int f \bar{g} h d m$.

\section{REFERENCES}

[1] V. M. Adamjan, D. Z. Arov and M. G. Krein, Infinite Hankel matrices and generalized Carathéodory-Fejér problems, Funct. Anal. Appl., 2 (1968), 1-19.

[2] R. Arocena and M. Cotlar, Generalized Toeplitz kernels and the Adamjan-ArovKrein moment problems, Toeplitz Centennial Volume, Operator Theory: Adv. \& Appl., 4 (1982), 37-55.

[3] R. Arocena, M. Cotlar and C. Sadosky, Weighted inequalities in $L^{2}$ and lifting properties, Advances in Math. Suppl. Studies 7A, 95-128, Academic Press, New York 1981.

[4] M. Cotlar and C. Sadosky, On the Helson-Szegö theorem and a related class of modified Toeplitz kernels, Proc. Symp. Pure Math. Amer. Math. Soc., 35:I (1979), 383-407. 
[5] M. Cotlar and C. Sadosky, On some $L^{p}$ versions of the Helson-Szegö theorem, Harmonic Analysis Conference in honour of Prof. A. Zygmund, Wadsworth Int. Math. Series (1982).

[6] _ A lifting theorem for subordinated invariant kernels, J. Funct. Anal., 67 (1986), 345-359.

[7] _ L Lifting properties, Nehari theorem and Paley lacunary inequality, Rev. Matem. Iberoamericana, 2 (1986), 55-71.

[8] P. Hartman, On completely continuous Hankel matrices, Proc. Amer. Math. Soc., 9 (1958), 362-366.

[9] H. Helson and D. Sarason, Past and future, Math. Scand., 21 (1967), 5-16.

[10] H. Helson and G. Szegö, A problem in prediction theory, Ann. Mat. Pura Appl., 51 (1960), 107-138.

[11] K. Hoffman, Banach Spaces of Analytic Functions, Englewood Cliffs, N.J. (1962).

[12] P. Koosis, Weighted quadratic means and Hilbert transforms, Duke Math. J., 38 (1971), 609-634.

[13] Z. Nehari, On bounded bilinear forms, Ann. of Math., 65 (1957), 153-162.

[14] L. B. Page, Applications of the Sz-Nagy and Foias lifting theorem, Indiana Univ. Math. J., 20 (1970), 135-145.

[15] S. C. Power, Hankel Operators on Hilbert Space (Research Notes in Math. Vol. 64), Pitman Advanced Publishing Program, Boston London Melbourne.

[16] F. Riesz and M. Riesz, Über Randverte einer analytischen Funktionen, Quatriéme Congres des Math. Scand., (1916), 27-44.

[17] T. Yamamoto, On the generalization of the theorem of Helson and Szegö, Hokkaido Math. J., 14 (1985), 1-11.

Received October 1, 1987 and in revised form September 26, 1990. This research was partially supported by Grant-in-Aid for Scientific Research, Ministry of Education, Japan.

HOKKAIDO UNIVERSITY

SAPPORO 060 JAPAN 



\section{PACIFIC JOURNAL OF MATHEMATICS EDITORS}

\author{
V. S. VARADARAJAN \\ (Managing Editor) \\ -University of California \\ Los Angeles, CA 90024-1555-05 \\ Herbert Clemens \\ University of Utah \\ Salt Lake City, UT 84112 \\ THOMAS ENRIGHT \\ University of California, San Diego \\ La Jolla, CA 92093
}

R. FINN

Stanford University

Stanford, CA 94305

Hermann FlaschKa

University of Arizona

Tucson, AZ 85721

VAUGHAN F. R. JONES

University of California

Berkeley, CA 94720

SteVen KerCKhoff

Stanford University

Stanford, CA 94305
C. C. MOORE

University of California

Berkeley, CA 94720

Martin ScharlemanN

University of California

Santa Barbara, CA 93106

\section{HAROLD STARK}

University of California, San Diego

La Jolla, CA 92093

\section{ASSOCIATE EDITORS}

\begin{tabular}{|c|c|c|c|c|}
\hline R. ARENS & $\begin{array}{l}\text { E. F. BECKENBACH } \\
(1906-1982)\end{array}$ & NeUManN & $\begin{array}{l}\text { F. WoLF } \\
(1904-1989)\end{array}$ & K. YoshidA \\
\hline \multicolumn{5}{|c|}{ SUPPORTING INSTITUTIONS } \\
\hline \multicolumn{2}{|c|}{ UNIVERSITY OF ARIZONA } & \multicolumn{3}{|c|}{ UNIVERSITY OF OREGON } \\
\hline \multirow{2}{*}{\multicolumn{2}{|c|}{$\begin{array}{l}\text { UNIVERSITY OF BRITISH COLUMBIA } \\
\text { CALIFORNIA INSTITUTE OF TECHNOLOGY }\end{array}$}} & \multicolumn{3}{|c|}{ UNIVERSITY OF SOUTHERN CALIFORNIA } \\
\hline & & \multicolumn{3}{|c|}{ STANFORD UNIVERSITY } \\
\hline CALIFORNIA INSTITUTE OF TECHNOLOGY & UNIVERSITY OF CALIFORNIA & \multicolumn{3}{|c|}{ UNIVERSITY OF HAWAII } \\
\hline \multicolumn{2}{|c|}{ MONTANA STATE UNIVERSITY } & \multicolumn{3}{|c|}{ UNIVERSITY OF TOKYO } \\
\hline \multicolumn{2}{|c|}{ UNIVERSITY OF NEVADA, RENO } & \multicolumn{3}{|c|}{ UNIVERSITY OF UTAH } \\
\hline \multicolumn{2}{|c|}{ NEW MEXICO STATE UNIVERSITY } & \multicolumn{3}{|c|}{ WASHINGTON STATE UNIVERSITY } \\
\hline OREGON STA & UNIVERSITY & UNIVERS & OF WASHING & \\
\hline
\end{tabular}




\section{Pacific Journal of Mathematics}

Vol. 150, No. $1 \quad$ September, 1991

Jan Aarts, Charles Lemuel Hagopian and Lex Gerard Oversteegen, The orientability of matchbox manifolds $\ldots \ldots \ldots \ldots \ldots \ldots \ldots \ldots \ldots \ldots \ldots \ldots$

Roger W. Barnard, Carl Hanson Fitzgerald and Sheng Gong, The growth and $1 / 4$-theorems for starlike mappings in $\mathbf{C}^{n} \ldots \ldots \ldots \ldots \ldots \ldots \ldots$

Allan Berele, Trace rings for verbally prime algebras $\ldots \ldots \ldots \ldots \ldots \ldots \ldots 23$

Quo-Shin Chi, Curvature characterization and classification of rank-one

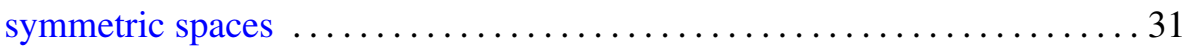

Harold Gerard Donnelly and Jeffrey Marc Lee, Domains in Riemannian

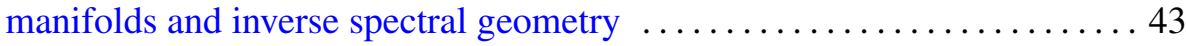

Clifford John Earle, Jr. and Patricia Lilaine Sipe, Families of Riemann

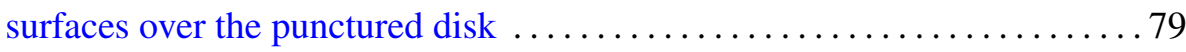

Mark P. Hughes, Dihedral group actions on homotopy complex projective

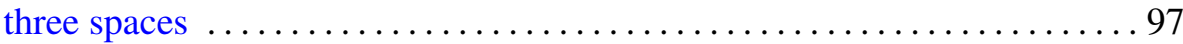

Robert F. Lax and Carl Widland, Gap sequences at a singularity ........111

Takahiko Nakazi, Bounded Hankel forms with weighted norms and lifting

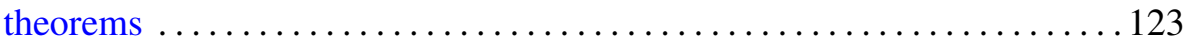

Douglas Murray Pickrell, Mackey analysis of infinite classical motion groups

Martha Rzedowski-Calderón and Gabriel Daniel Villa-Salvador,

Automorphisms of congruence function fields 167

Peter N-S Wong, Equivariant Nielsen fixed point theory for $G$-maps 